\title{
Optimal indications and limitations of endoscopic transorbital superior eyelid surgery for spheno-orbital meningiomas
}

\author{
Doo-Sik Kong, MD, PhD, ${ }^{1}$ Yong Hwy Kim, MD, PhD, ${ }^{2}$ and Chang-Ki Hong, MD, PhD ${ }^{3}$ \\ 1Department of Neurosurgery, Samsung Medical Center, Sungkyunkwan University School of Medicine; ²Department of \\ Neurosurgery, Seoul National University College of Medicine; and ' ${ }^{2}$ epartment of Neurosurgery, Gangnam Severance Hospital, \\ Yonsei University Health System, Seoul, Republic of Korea
}

\begin{abstract}
OBJECTIVE Spheno-orbital meningiomas (SOMs) are complicated tumors that involve multiple structures at initial presentation, such as the orbit, temporalis muscle, sphenoidal bone, cavernous sinus, and temporal or infratemporal fossa. The infiltrative growth and complexity of this type of meningioma make total resection impossible. In this study, the authors evaluated the surgical outcome of the endoscopic transorbital approach (eTOA) for SOM. In addition, they identified optimal indications for the use of eTOA and analyzed the feasibility of this approach as a minimally invasive surgery for SOMs of varying types and locations at presentation.
\end{abstract}

METHODS Between September 2016 and December 2019, the authors performed eTOA in 41 patients with SOM with or without orbital involvement at 3 independent tertiary institutions. The authors evaluated the surgical outcomes of eTOA for SOM and investigated several factors that affect the outcome, such as tumor volume, tumor location, and the presence of lateral orbitotomy. Gross-total resection (GTR) was defined as complete resection of the tumor or intended subtotal resection except the cavernous sinus. This study was undertaken as a multicenter project (006) of the Korean Society of Endoscopic Neurosurgery (KOSEN-006).

RESULTS There were 41 patients ( 5 men and 36 women) with a median age of 52.0 years (range 24-73 years). Twentyone patients had tumors that involved the orbital structure, while 14 patients had tumors that presented at the sphenoidal bone along with other structures, such as the cavernous sinus, temporal fossa, and infratemporal fossa. Fifteen patients had the globulous type of tumor and 26 patients had the en plaque type. Overall, GTR was achieved in 21 of 41 patients (51.2\%), and complications included CSF leaks in 2 patients and wound complications in 2 patients. Multiple logistic regression analysis showed that the en plaque type of tumor, absence of lateral orbital rim osteotomy, involvement of the temporal floor or infratemporal fossa, and involvement of the orbit and medial one-third of the greater sphenoidal wing were closely associated with lower GTR rates $(p<0.05)$. Multivariate analysis revealed that the en plaque type of tumor and the absence of lateral orbital rim osteotomy were significant predictors for lower GTR rate.

CONCLUSIONS The en plaque type of SOM remains a challenge despite advances in technique such as minimally invasive surgery. Overall, clinical outcome of eTOA for SOM was comparable to the transcranial surgery. To achieve GTR, ETOA is recommended, with additional lateral orbital rim osteotomy for globulous-type tumors, without involving the floor of the temporal and infratemporal fossa.

https://thejns.org/doi/abs/10.3171/2020.3.JNS20297

KEYWORDS endoscopic transorbital approach; spheno-orbital meningioma; en plaque type; skull base

$\mathrm{M}$ ENINGIOMAS involving the skull base remain challenging because of their proximity to surrounding critical neurovascular structures. Thus, a wide exposure of bones and muscles is required to facilitate safe and complete surgical resection of these tumors. Among them, spheno-orbital meningiomas (SOMs) have a high incidence of meningeal en plaque growth patterns with hyperostotic bony changes, which occasionally result in the direct compression of surrounding structures such as the orbit, cavernous sinus, or infratemporal areas..$^{1,2}$ To date, surgical management of these tumors has traditionally been achieved by transcranial approaches, i.e., the pterional approach, frontotemporal approach, and frontotemporal orbitozygomatic approach, ${ }^{1}$ which sometimes

ABBREVIATIONS ETOA = endoscopic transorbital approach; GTR = gross-total resection; SOM = spheno-orbital meningioma.

SUBMITTED January 31, 2020. ACCEPTED March 30, 2020.

INCLUDE WHEN CITING Published online June 5, 2020; DOI: 10.3171/2020.3.JNS20297. 
result in large skull base defects with cosmetic issues such as bony depression, atrophy of the temporalis muscle, and a long skin scar behind the hairline after surgery. Nevertheless, these tumors cannot be completely removed with conventional transcranial surgeries because of their innate characteristics, such as a highly infiltrative nature and greater tumor complexity. Through significant advances in modern surgical techniques and recent developments in radiosurgery and conformal radiotherapeutic modalities, the surgical therapeutic goal for these complex tumors has been modified from complete resection to minimally invasive and safe resection followed by radiosurgery or radiotherapy.

Recently, the endoscopic transorbital approach (eTOA) with a superior eyelid incision has emerged as a minimally invasive technique, which introduces a lateral approach to the orbit. ${ }^{2-6}$ This approach can provide an additional surgical corridor into the anterior and middle cranial fossas and has the following advantages: it reduces the scar length, prevents excessive retraction of the temporalis muscle, and leaves a smaller cranial bone defect. However, it is still unknown which types of meningiomas can be optimally treated with this novel surgical approach. In this study, we collected preliminary data regarding the use of eTOA for SOM and analyzed the surgical outcomes and adverse events. Additionally, we identified the optimal indications for and surgical pitfalls of this novel approach.

\section{Methods}

Between September 2016 and December 2019, we analyzed 41 patients with SOM who underwent eTOA at 3 independent tertiary institutions (23 patients at Samsung Medical Center, 17 patients at Gangnam Severance Hospital, and 1 patient at Seoul National University Hospital, Seoul, Korea). The study was undertaken as a multicenter project (006) of the Korean Society of Endoscopic Neurosurgery (KOSEN-006). Preoperative orbital manifestations included proptosis (20 patients), visual impairment (3 patients), and diplopia due to ocular paresis (8 patients). Seven patients had facial sensory changes. There were no definitive neurological or cranial nerve deficits (other than optic nerve) in all patients. In this study, surgical treatment was recommended when patients had neurological or orbital manifestations or the length of the tumor diameter exceeded $2 \mathrm{~cm}$, regardless of the presence of symptoms. Indications for eTOA included the following: 1) location of the epicenter of the tumor below the superior margin of the sphenoidal ridge, 2) long diameter of the tumor less than $6 \mathrm{~cm}$, and 3) no tumor involvement of the anterior clinoid process or middle cerebral arteries. All surgeries were performed by 3 neurosurgeons (D.K., Y.K., and C.H.) in collaboration with oculoplastic surgeons. Medical records and tumor registries of the patients were reviewed for preoperative radiological findings and several independent variables such as demographic data, the presence of orbital symptoms, tumor volume, tumor involvement, history of recurrence, and surgical outcomes and morbidities. The study was approved by the institutional review board and conducted in accordance with the ethical guidelines of the Declaration of Helsinki; the informed consent re- quirement was waived. Data for 12 of the patients were previously published with the authors' previous studies for analysis with respect to ophthalmological outcome. ${ }^{6}$

\section{Classification of Meningioma}

In this study, we defined a SOM as a subset of sphenoid wing meningioma mainly involving the lesser sphenoidal wing and causing pronounced hyperostosis with involvement of the greater sphenoid wing, orbit, and central skull base. $^{7}$ Based on preoperative T1-weighted contrast-enhanced MRI studies, we classified all tumors according to the following 3 types based on the location of the tumor epicenter on the greater sphenoidal bone: the medial onethird, middle one-third, and lateral one-third of the greater sphenoidal wing (Fig. 1). Further, depending on their growth pattern, the tumors were classified as en plaque type or globulous type. ${ }^{8,9}$ The globulous type of meningioma was defined as globulous in shape, with a short dural tail and a growth pattern with less infiltration to the surrounding organ or tissues than the en plaque type. In contrast, the en plaque type of meningioma was defined as relatively flat in shape, with a long dural tail and a growth pattern that was highly infiltrative to the surrounding tissues, such as the orbit, cavernous sinus, and surrounding osseous tissue. ${ }^{9}$ Additionally, we identified the preoperative involvement of the orbit, cavernous sinus, temporal and infratemporal fossas, and anterior clinoid process. All of these classifications were preoperatively performed by 2 independent neuroradiologists (S. T. Kim and I. K. Kim). We assessed the tumor volume with the Osirix free software program. Postoperatively, we analyzed the clinical outcome and gross-total resection (GTR) rate and further investigated the location of the remaining tumor after subtotal resection.

\section{Surgical Technique}

The technique of eTOA has already been described in detail in the previous literature..$^{4,10-12}$ Briefly, under general anesthesia, the patient was placed in the supine position with either a slight flexion of the head or the head in the neutral position. A skin incision was made along the superior eyelid crease after the induction of local anesthesia. After the skin-orbicularis muscular layer was exposed, suborbicularis dissection was simultaneously performed superolaterally to raise the skin-muscle flap to avoid injury to the underlying orbital septum and the levator palpebrae muscle. Once the lateral orbital rim was palpated, the periosteum was elevated along the superolateral orbital wall. In most cases, the greater sphenoidal wing consisting of the lateral orbital wall had a thickened and hypertrophic change induced by the tumor. Since pneumatic (Mitaka) and electrical (Endofix) endoscopic holding systems were introduced to our institutes, these holders have been used to facilitate endoscopic procedures after exposure of the lateral orbital rim. The greater sphenoidal wing was drilled gradually to expose the temporalis muscle, which is a key anatomical structure that provides good surgical orientation under endoscopic views. Decisions regarding whether to perform a lateral orbital rim osteotomy were made according to the lateral extent of the tumor. Lateral orbital 
rim osteotomy allowed increased surgical maneuverability to the lateral extent of the tumor, along with good visualization. Superior and inferior orbital fissures were additional relevant anatomical landmarks. Bone drilling of the greater sphenoidal wing and lesser sphenoidal bone was performed from the lateral to the medial direction. The anterior surface of the temporal dura was gradually exposed and opened with a knife under neuronavigation guidance. The extent of the dural incision was adjusted according to the extent of tumor involvement. In most cases, the tumor was debulked with the help of an ultrasonic aspirator or by simple suction alone. In tumors involving the ocular structures, the periorbital layer was decompressed and distinctly identified; however, in some cases, the periorbita and extraocular muscles, such as the lateral rectus muscle, were infiltrated by an en plaque type of tumor. In such cases, it is necessary to preserve the extraocular muscle to avoid permanent diplopia. For tumors extending to the temporal floor or infratemporal fossa, increased forward inclination of the head, a wider skin incision, and lateral orbital rim osteotomy might be helpful; however, in our experience surgical maneuverability remained limited even after with these modifications. At the completion of surgery, free fat tissue from the abdomen was harvested and grafted to fill the empty space intradurally. Buttonlayered fascia lata or allogenic dermis was grafted on the dura inlay and onlay. Next, free fat tissue was placed to fill the empty extradural space. Finally, an artificial bone plate (TnR mesh, 3D-printed bioresorbable mesh, T\&R biofab) was placed to cover the periosteum to prevent postoperative enophthalmos. The periosteum, subcutaneous tissue, and skin were sutured layer by layer.

\section{Statistical Analysis}

In this study, we defined GTR as a complete resection of the tumor or intended subtotal resection except in a lesion involving a cavernous sinus. Statistical analysis was performed using R software version 3.5. Multiple logistic regression analyses were performed to determine the variables that were independently associated with the GTR rate or intended subtotal resection. In the univariate regression model, variables that met a $\mathrm{p}$ value $<0.1$ were selected for multivariate analysis. Those variables in the multivariate regression analysis that had a $\mathrm{p}$ value $<0.05$ were determined to be statistically significant.

\section{Results \\ Demographic Data}

The demographic and clinical data are summarized in Table 1 . In this study cohort, women were predominant (36 women vs 5 men). The median patient age was 52.0 years (range 24-73 years). Thirty-four patients had a primary tumor and 7 patients had a recurrent tumor (6 patients had previously undergone transcranial surgery and 1 patient had received radiosurgery). The mean tumor volume was $20.8 \mathrm{~cm}^{3}$ (range $4.6-81.3 \mathrm{~cm}^{3}$ ), the mean resection volume was $18.2 \mathrm{~cm}^{3}$ (range $3.5-50.0 \mathrm{~cm}^{3}$ ), and the mean degree of resection was $83.9 \%$ (46.0\%-100\%). Histologically, all meningiomas, except 2 atypical meningiomas, were benign types such as meningothelial or transitional type.

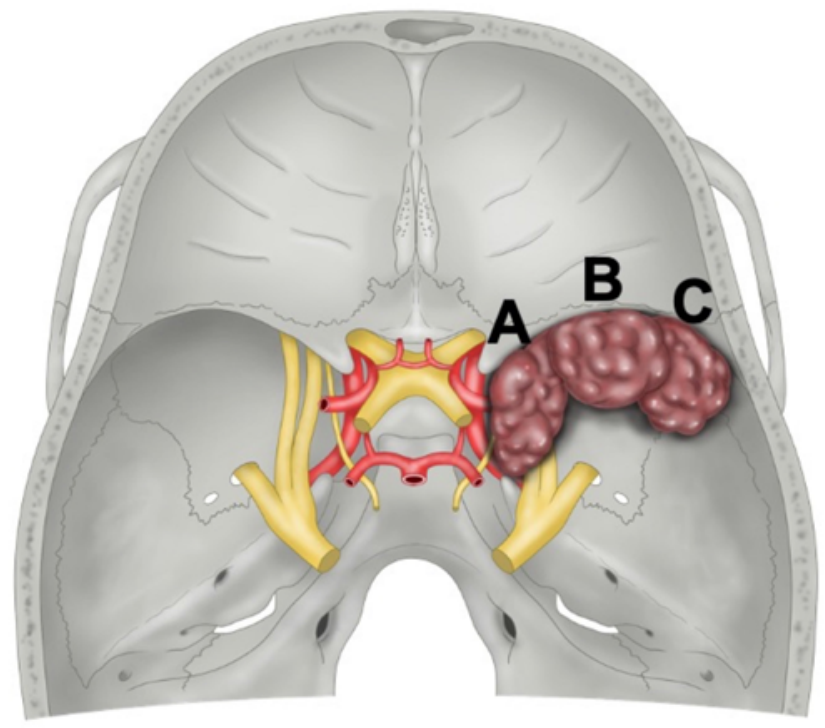

FIG. 1. Illustration of a SOM with the location of the tumor epicenter on the greater sphenoidal bone: the medial one-third $(\mathbf{A})$, middle one-third (B), and lateral one-third (C) of the greater sphenoidal wing. (C) 2000 Samsung Medical Center, Seoul, Korea. Used with permission.

\section{Extracranial Involvement}

Overall, 28 of 41 patients (68.3\%) had tumors involving extracranial compartments such as the orbit, cavernous sinus, and temporal and infratemporal fossas, and 23 of 41 patients had accompanying sphenoidal bone hypertrophy. In 21 patients, the tumor involved orbital structures such as the periorbita (5 patients) and/or extraocular muscles (16 patients). Fifteen patients had a tumor involving the cavernous sinus without any neurological symptoms or signs. The floor of the temporal fossa was involved in 18 patients, and in 9 of these patients the tumor extended to the infratemporal fossa. Overall, 14 patients showed a tumor involving 2 or more extracranial compartments.

\section{Intracranial Involvement}

Regarding tumor location, the tumor epicenter was at the medial one-third of the sphenoidal wing in 15 patients, at the middle one-third in 10 patients, at the lateral onethird in 4 patients, and involved the entire sphenoidal bone in 12 patients. There were $15(36.6 \%)$ patients with the globulous type of tumor either with or without sphenoidal hypertrophic change and 26 (63.4\%) patients with the en plaque type of tumor.

\section{Clinical Outcome}

The mean follow-up period was 15.9 months. Overall, complete resection was achieved in 17 of 41 patients (41.5\%, Fig. 2 and Video 1).

VIDEO 1. A 40-year-old female patient with proptosis and paresthesia on the right side of her face was diagnosed with SOM without involvement of the cavernous sinus, orbit, or infratemporal fossa. She underwent eTOA surgery for tumor and GTR was performed, as shown in this video. (C) 2000 Samsung Medical Center, Seoul, Korea. Used with permission. Click here to view. 
TABLE 1. Patient characteristics and clinical outcomes

\begin{tabular}{|c|c|}
\hline & Value \\
\hline Total no. of patients & 41 \\
\hline \multicolumn{2}{|l|}{ Sex } \\
\hline Male & 5 \\
\hline Female & 36 \\
\hline Age, yrs & $52.0(24-73)$ \\
\hline \multicolumn{2}{|l|}{ Meningioma histopathology } \\
\hline Benign (grade I) & 39 \\
\hline Atypical (grade II) & 2 \\
\hline \multicolumn{2}{|l|}{ Primary vs recurrent tumor } \\
\hline Primary & 34 \\
\hline Recurrent & 7 \\
\hline Tumor vol, $\mathrm{cm}^{3}$ & $20.8(4.6-81.3)$ \\
\hline Resection vol, $\mathrm{cm}^{3}$ & $18.2(3.5-50.0)$ \\
\hline Degree of resection, $\%$ & $83.9(46.0-100)$ \\
\hline \multicolumn{2}{|l|}{ Extracranial involvement } \\
\hline Hypertrophic change of sphenoidal bone & 23 \\
\hline Orbit involvement & 21 \\
\hline Cavernous sinus & 15 \\
\hline Temporal floor or infratemporal fossa & 18 \\
\hline \multicolumn{2}{|l|}{ Intracranial involvement } \\
\hline \multicolumn{2}{|l|}{ Tumor epicenter (sphenoid) } \\
\hline Medial $1 / 3$ & 15 \\
\hline Middle 1/3 & 10 \\
\hline Lateral $1 / 3$ & 4 \\
\hline Whole compartment & 12 \\
\hline \multicolumn{2}{|l|}{ Growth pattern } \\
\hline Globulous & 15 \\
\hline En plaque & 26 \\
\hline Mean follow-up period, mos & 15.9 \\
\hline \multicolumn{2}{|l|}{ Extent of resection } \\
\hline GTR & 21 \\
\hline Gross-total removal & 17 \\
\hline $\begin{array}{l}\text { Gross-total removal except cavernous sinus } \\
\text { portion }\end{array}$ & 4 \\
\hline Subtotal or partial resection & 20 \\
\hline Lateral orbital rim osteotomy & 22 \\
\hline Mean op time, hrs & 4.6 \\
\hline \multicolumn{2}{|l|}{ Postop morbidities } \\
\hline CSF leak & 2 \\
\hline Wound complication & 2 \\
\hline \multicolumn{2}{|l|}{ Postop radiotherapy } \\
\hline Gamma Knife radiosurgery & 13 \\
\hline
\end{tabular}

Values are presented as number of patients, mean, or median (range).

In 4 patients, subtotal resection was intended because of the involvement of the cavernous sinus area. The goal of intended subtotal resection was to resect all tumors except lesions involving the cavernous sinus, to avoid new neurological deficits. Thus, 21 of 41 patients $(51.2 \%)$ underwent GTR in this study by definition. In 20 patients, only subtotal resection was achieved because of inability to reach the area involving the lateral margin of the tumor or the presence of a lower margin such as the temporal floor or infratemporal fossa (Fig. 3). GTR was performed for 21 tumors that involved the orbital structure and 17 of 27 tumors that involved the temporal floor or infratemporal fossa. To obtain a wide working space and increased surgical maneuverability, a lateral orbital rim osteotomy was performed in 22 patients. The median hospitalization duration was 4 days (3-30 days). Postoperatively, proptosis was completely or partially improved in all 20 affected patients and visual function was restored in 2 of 3 patients with preoperative visual impairment.

\section{Postoperative Morbidities}

Postoperative morbidities included CSF leak in 2 patients, which resolved with lumbar drainage of CSF for 3 days, and wound complications in 2 patients. One wound complication was keloid scar formation, which improved when treated with intralesional steroid therapy, and the other was skin infection, which required further intravenous antibiotic treatment for 2 weeks. There were no cases of postoperative mortality, diplopia, permanent ptosis, or enophthalmos during the follow-up period. Overall, the cosmetic outcomes seemed satisfactory (Fig. 4). Among the patients with residual tumor after surgery, 13 patients were treated with Gamma Knife radiosurgery for tumor growth control.

\section{Prognostic Factors Affecting the Clinical Outcome}

To identify the prognostic factors affecting GTR (complete resection or intended subtotal resection) of SOM, several independent variables, such as sex, age, the presence of cavernous invasion, tumor volume, involvement of the temporal floor or infratemporal fossa, recurrence, lateral orbital rim osteotomy, location of tumor epicenter, orbital involvement, and growth pattern such as en plaque or globulous type, were analyzed. First, for complete resection alone, univariate and multivariate regression analyses showed that the en plaque type of meningioma $(\mathrm{p}<0.01)$ and cavernous invasion $(\mathrm{p}<0.01)$ were significant prognostic factors. Second, according to the new concept of GTR (complete resection or intended subtotal resection), we excluded the presence of cavernous sinus invasion from the independent variables. In this situation, univariate regression analysis showed that with the enplaque meningioma pattern in the absence of lateral orbital rimosteotomy, the involvement of the temporal floor or infratemporal fossa and the involvement of the orbit and the medial one-third of the greater sphenoidal wing were closely associated with lower GTR rates $(\mathrm{p}<0.05$, Table 2). Specifically, the en plaque type of meningioma had a lower GTR rate $(11.5 \%)$, whereas the globulous type had a higher GTR rate $(93.3 \%$; $p<0.01)$. Patients who underwent lateral orbital rim osteotomy had a higher resection rate than those without lateral orbital rim removal $(54.5 \%$ vs $26.3 \%$; $p<0.01$ ). Tumors with involvement of the temporal floor or infratemporal fossa, orbit, and medial onethird of the greater sphenoidal wing showed worse resection rates than those without such involvements $(16.7 \%$ 

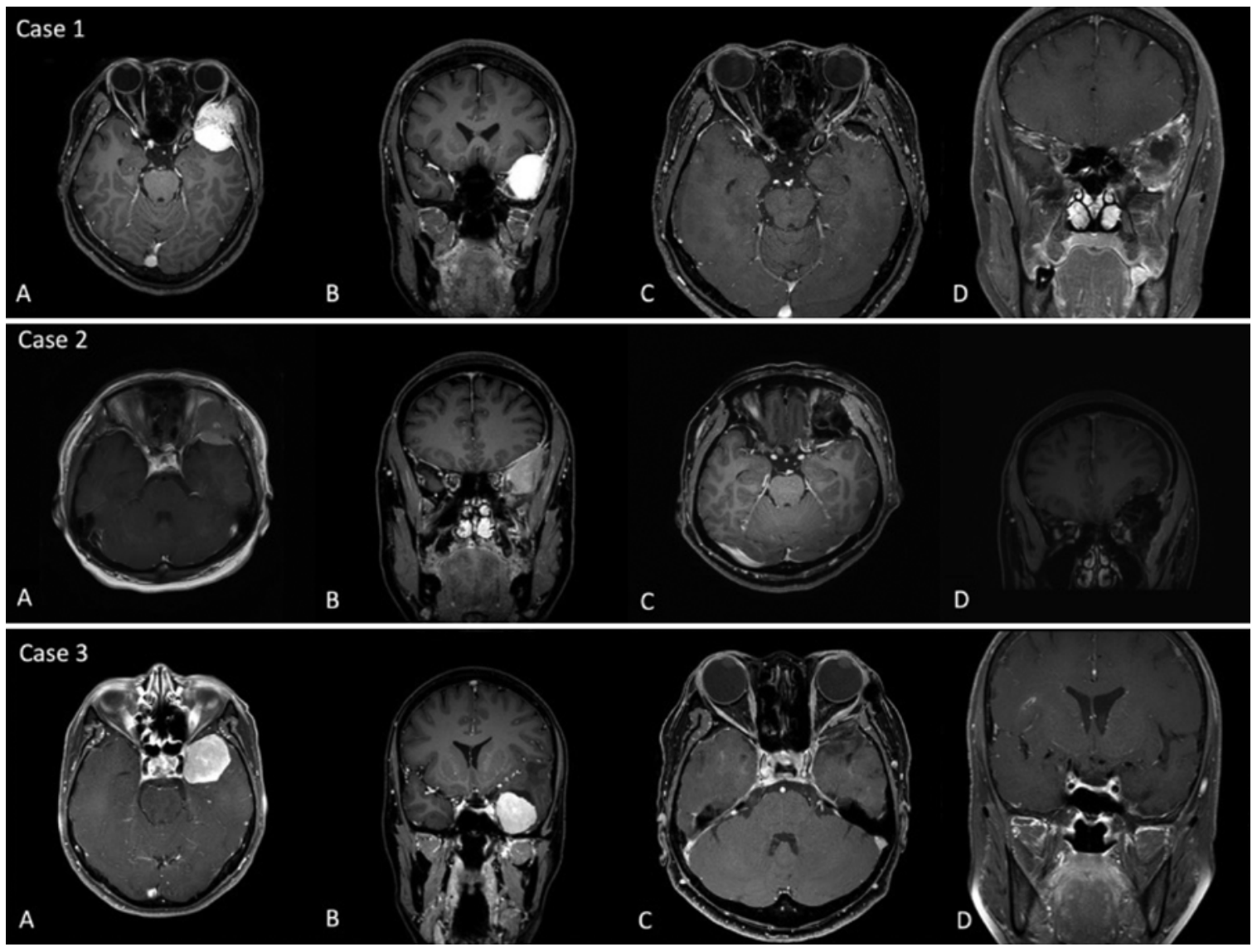

FIG. 2. Preoperative (A and B) and postoperative (C and D) axial and coronal MR images after GTR (cases 1, 2, and 3). Case 1, globulous type of SOM involving the middle and lateral one-third of the greater sphenoidal wing; case 2, globulous type of meningioma involving the orbit and lateral one-third of the greater sphenoidal wing; and case 3, globulous type of meningioma involving the whole segment of the greater sphenoidal wing.

vs $60.9 \%, 19.0 \%$ vs $65.0 \%$, and $18.5 \%$ vs $85.7 \%$, respectively). To determine the variables that were independently associated with the resection rate, we performed a multivariate regression analysis using the 5 variables found to be statistically significant in the univariate analyses $(\mathrm{p}<$ $0.1)$. Multivariate analysis demonstrated that the en plaque type and the absence of lateral orbital rim osteotomy were significant predictors for lower GTR rate $(\mathrm{p}<0.01)$.

\section{Discussion}

SOM arises from the dura mater overlying the greater or lesser sphenoid wing and represents approximately $9 \%$ of all intracranial meningiomas. ${ }^{1,2,13-15}$ These tumors frequently show a meningeal en plaque growth pattern and accompany hyperostotic bony changes. ${ }^{1,2,8,9}$ In our study, the en plaque type SOM was prevalent ( $>70 \%$ of cases). The spreading pattern of this type of meningioma makes total resection impossible. Therefore, it is essential to identify the final goal of surgical treatment for SOM; either complete resection or intracranial decompression followed by radiosurgery or radiotherapy may be selected. The multicompartmental involvement of this complex tumor requires an excessive downward retraction of the temporalis muscle and extensive drilling of the squamous temporal bone and the greater sphenoidal bone, which results in a distinct scar, deformity of the temporal bone, and permanent atrophic changes of the temporalis muscle. Such serious cosmetic issues have resulted in a need for minimally invasive surgical techniques for the removal of this deep-seated lesion.

The retro-orbital corridor provides a potential route to reach the anterior and middle cranial fossa in a minimally invasive fashion. ${ }^{1,12}$ However, overcrowding with surgical instruments in this narrow corridor is a challenge for surgeons. The main concern regarding the eTOA is that it carries a risk of probable orbital injury and bradycardia due to activation of the oculocardiac reflex during excessive retraction of the orbital globe. With recent improvements in surgical instruments and techniques, the endo/exo-orbital corridor has emerged as a viable surgical option for endoscopic neurosurgery.

\section{Study Limitations: Surgical Maneuverability}

Although eTOA is a minimally invasive technique for deep-seated orbital and skull base lesions, surgical maneuverability in a narrow surgical corridor remains a challenge. ${ }^{1,12,16-28}$ To gain surgical freedom during eTOA, a limited lateral orbital rim osteotomy may be helpful. ${ }^{16,29}$ 

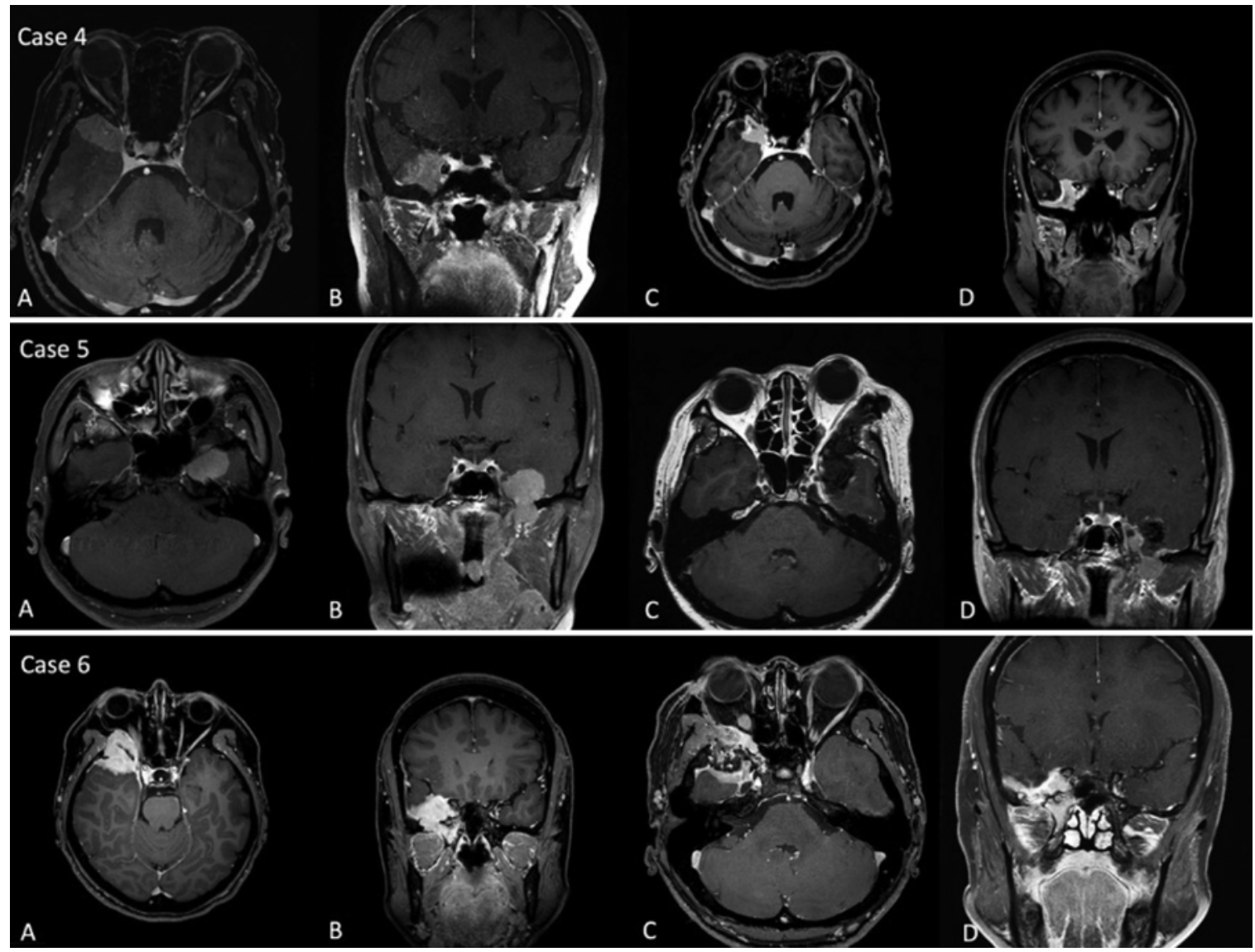

FIG. 3. Preoperative (A and B) and postoperative ( $C$ and $\mathbf{D}$ ) axial and coronal MR images after subtotal resection of tumor (cases 4,5 , and 6). Case 4, SOM involving the temporal floor, infratemporal fossa, and cavernous sinus; case 5, meningioma involving the medial one-third of the greater sphenoidal wing and extending into the infratemporal fossa; case 6 , en plaque type of meningioma involving the orbit, cavernous sinus, temporal floor, and infratemporal fossa.
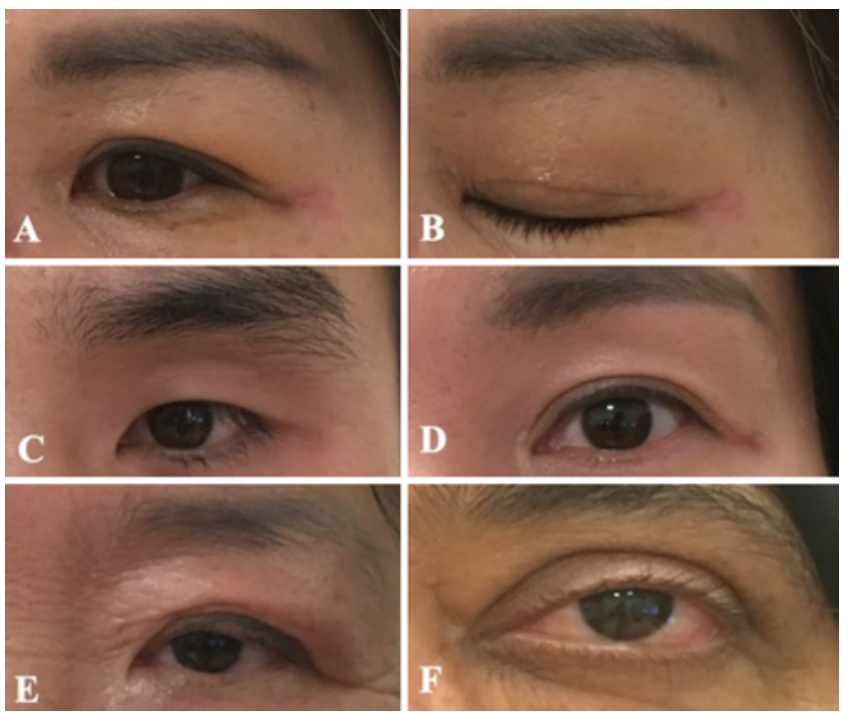

FIG. 4. Postoperative patient photographs showing cosmetic results 2-6 months after eTOA surgery. A and B: A 47-year-old female patient with the eye open (A) and closed (B), who underwent additional lateral orbital rim osteotomy. C: A 41-year-old male patient with the eye open. D: A 44-year-old female patient with the eye open. E: A 60-year-old female patient with the eye open, who experienced immediate eyelid swelling from wound infection. F: A 59-year-old female patient with the eye open.
Lateral orbital osteotomy is familiar to orbital surgeons, and detachment of the temporalis muscle fascia from the lateral orbital rim should be achieved up to the lateral canthal tendon. The extent of lateral orbital osteotomy starts with the frontozygomatic suture above and includes the base of the lateral orbital rim below. This simple procedure can facilitate wider maneuverability and enables GTR. In this study, we additionally performed lateral orbital osteotomy in cases of SOM involving the lateral one-third of the greater sphenoidal wing, which resulted in improvement of the resection rate. We also found that tumors involving the temporal floor and infratemporal fossa were difficult to remove through the transorbital route. Because of concern that the main surgical corridor of eTOA is directed toward the superolateral part of the middle cranial fossa, ${ }^{18,25,27}$ enhancing the technique to overcome this limitation is an essential goal.

Based on this study, the fact that achieving GTR of SOM is still a challenge should be taken into consideration despite the increased safety and decreased invasiveness of minimally invasive endoscopic surgery. This difficulty is attributable to the geographical complexity of SOM and the narrow surgical corridor. Moreover, it should be noted that the eTOA technique is still unfamiliar to most surgeons regardless of their experience in the neurosurgical 
TABLE 2. Logistic regression analysis for factors predictive of GTR (complete or intended subtotal removal) for SOMs

\begin{tabular}{|c|c|c|c|c|c|c|}
\hline \multirow[b]{3}{*}{ Variable } & \multicolumn{6}{|c|}{ Analysis } \\
\hline & \multicolumn{3}{|c|}{ Univariable } & \multicolumn{3}{|c|}{ Multivariable } \\
\hline & $\mathrm{p}$ Value & OR & $95 \% \mathrm{Cl}$ & $\mathrm{p}$ Value & OR & $95 \% \mathrm{Cl}$ \\
\hline Globulous type & 0.00 & 38.00 & $4.19-345.04$ & 0.00 & 71.90 & $4.57-1131.18$ \\
\hline Tumor vol >20 $\mathrm{cm}^{3}$ & 0.77 & 0.78 & $0.14-4.27$ & - & - & - \\
\hline Lateral orbital rim osteotomy & 0.00 & 7.47 & $1.87-29.88$ & 0.02 & 15.79 & $1.64-151.73$ \\
\hline Recurrent tumor & 0.20 & 0.32 & $0.05-1.86$ & - & - & - \\
\hline \multicolumn{7}{|l|}{ Tumor involvement } \\
\hline Temporal floor or infratemporal fossa & 0.01 & 5.94 & $1.52-23.18$ & - & - & - \\
\hline Orbit & 0.02 & 4.67 & $1.23-17.44$ & - & - & - \\
\hline Medial $1 / 3$ of greater sphenoidal wing & 0.00 & 0.08 & $0.02-0.46$ & - & - & - \\
\hline
\end{tabular}

field. Therefore, a thorough understanding of the complex anatomy around the orbit through dissection of cadaver specimens and acquired experience in transorbital surgery will allow surgeons to perform maximal safe resection with minimally invasive access via the orbital route.

\section{Conclusions}

Overall, the clinical outcomes of patients undergoing eTOA for SOM were comparable to those for transcranial surgery. The eTOA with additional lateral orbital rim osteotomy is recommended to achieve GTR of the globulous type of tumor, which does not involve the floor of the temporal and infratemporal fossas.

\section{References}

1. De Rosa A, Pineda J, Cavallo LM, et al. Endoscopic endoand extra-orbital corridors for spheno-orbital region: anatomic study with illustrative case. Acta Neurochir (Wien). 2019;161(8):1633-1646.

2. Dallan I, Sellari-Franceschini S, Turri-Zanoni M, et al. Endoscopic transorbital superior eyelid approach for the management of selected spheno-orbital meningiomas: preliminary experience. Oper Neurosurg (Hagerstown). 2018;14(3):243251.

3. Dallan I, Locatelli D, Turri-Zanoni M, et al. Transorbital endoscopic assisted resection of a superior orbital fissure cavernous haemangioma: a technical case report. Eur Arch Otorhinolaryngol. 2015;272(12):3851-3856.

4. Moe KS, Kim LJ, Bergeron CM. Transorbital endoscopic repair of cerebrospinal fluid leaks. Laryngoscope. 2011;121(1):13-30.

5. Jeon C, Hong CK, Woo KI, et al. Endoscopic transorbital surgery for Meckel's cave and middle cranial fossa tumors: surgical technique and early results. J Neurosurg. 2019;131(4):1126-1135.

6. Kong DS, Young SM, Hong CK, et al. Clinical and ophthalmological outcome of endoscopic transorbital surgery for cranioorbital tumors. J Neurosurg. 2018;131(3):667-675.

7. Kiyofuji S, Casabella AM, Graffeo CS, et al. Sphenoorbital meningioma: a unique skull base tumor. Surgical technique and results. J Neurosurg. Published online August 23, 2019. doi:10.3171/2019.6.JNS191158

8. Park HH, Yoo J, Yun IS, Hong CK. Comparative analysis of endoscopic transorbital approach and extended minipterional approach for sphenoid wing meningiomas with osseous involvement: preliminary surgical results. World Neurosurg. Published online January 27, 2020. doi:10.1016/j. wneu.2020.01.115

9. Roser F, Nakamura M, Jacobs C, et al. Sphenoid wing meningiomas with osseous involvement. Surg Neurol. 2005;64(1):37-43.

10. Noiphithak R, Yanez-Siller JC, Revuelta Barbero JM, et al. Comparative analysis of the exposure and surgical freedom of the endoscopic extended minipterional craniotomy and the transorbital endoscopic approach to the anterior and middle cranial fossae. Oper Neurosurg (Hagerstown). 2019;17(2):174-181.

11. Dallan I, Caniglia M, Turri-Zanoni M, et al. Transorbital superior eyelid endoscopic approach to the temporal lobe. $J$ Neurosurg Sci. 2018;62(3):369-372.

12. Di Somma A, Andaluz N, Cavallo LM, et al. Endoscopic transorbital superior eyelid approach: anatomical study from a neurosurgical perspective. J Neurosurg. 2018;129(5):12031216.

13. Freeman JL, Davern MS, Oushy S, et al. Spheno-orbital meningiomas: a 16-year surgical experience. World Neurosurg. 2017:99:369-380.

14. Honig S, Trantakis C, Frerich B, et al. Meningiomas involving the sphenoid wing outcome after microsurgical treatment-a clinical review of 73 cases. Cent Eur Neurosurg. 2010;71(4):189-198.

15. Almeida JP, Omay SB, Shetty SR, et al. Transorbital endoscopic eyelid approach for resection of sphenoorbital meningiomas with predominant hyperostosis: report of 2 cases. $J$ Neurosurg. 2018;128(6):1885-1895.

16. Alqahtani A, Padoan G, Segnini G, et al. Transorbital transnasal endoscopic combined approach to the anterior and middle skull base: a laboratory investigation. Acta Otorhinolaryngol Ital. 2015;35(3):173-179.

17. Allen RC. The evolving role of the oculoplastic surgeon in skull base surgery. Curr Opin Ophthalmol. 2016;27(5):420427.

18. Gerges MM, Godil SS, Younus I, et al. Endoscopic transorbital approach to the infratemporal fossa and parapharyngeal space: a cadaveric study. J Neurosurg. Published online November 1, 2019. doi:10.3171/2019.7.JNS191743

19. Balakrishnan K, Moe KS. Applications and outcomes of orbital and transorbital endoscopic surgery. Otolaryngol Head Neck Surg. 2011;144(5):815-820.

20. Dallan I, Castelnuovo P, Turri-Zanoni M, et al. Transor- 
bital endoscopic assisted management of intraorbital lesions: lessons learned from our first 9 cases. Rhinology. 2016;54(3):247-253.

21. Golbin DA, Lasunin NV, Cherekaev VA, et al. Biopsy and resection of skull base tumors using transorbital endoscopic approaches: primary results. Article in Russian. Vopr Neirokhir. 2019;83(3):42-56.

22. Lee MH, Hong SD, Woo KI, et al. Endoscopic endonasal versus transorbital surgery for middle cranial fossa tumors: comparison of clinical outcomes based on surgical corridors. World Neurosurg. 2019;122:e1491-e1504.

23. Lin BJ, Hong KT, Chung TT, et al. Endoscopic transorbital transtentorial approach to middle incisural space: preclinical cadaveric study. Acta Neurochir (Wien). 2019;161(4):831-839.

24. Lin BJ, Ju DT, Hsu TH, et al. Endoscopic transorbital approach to anterolateral skull base through inferior orbital fissure: a cadaveric study. Acta Neurochir (Wien). 2019;161(9):1919-1929.

25. Locatelli D, Pozzi F, Turri-Zanoni M, et al. Transorbital endoscopic approaches to the skull base: current concepts and future perspectives. J Neurosurg Sci. 2016;60(4):514-525.

26. Park HH, Hong SD, Kim YH, et al. Endoscopic transorbital and endonasal approach for trigeminal schwannomas: a retrospective multicenter analysis (KOSEN-005). J Neurosurg Published online June 21, 2019. doi:10.3171/2019.3.JNS19492

27. Priddy BH, Nunes CF, Beer-Furlan A, et al. A side door to Meckel's cave: anatomic feasibility study for the lateral transorbital approach. Oper Neurosurg (Hagerstown). 2017;13(5):614-621.

28. Noiphithak R, Yanez-Siller JC, Revuelta Barbero JM, et al. Quantitative analysis of the surgical exposure and surgical freedom between transcranial and transorbital endoscopic anterior petrosectomies to the posterior fossa. J Neurosurg. 2018;131(2):569-577.

29. Tai AX, Sack KD, Herur-Raman A, Jean WC. The benefits of limited orbitotomy on the supraorbital approach: an anatomic and morphometric study in virtual reality. Oper Neurosurg (Hagerstown). 2020;18(5):542-550.

\section{Disclosures}

The authors report no conflict of interest concerning the materials or methods used in this study or the findings specified in this paper.

\section{Author Contributions}

Acquisition of data: Kong, Kim. Analysis and interpretation of data: Kong. Drafting the article: Kong. Critically revising the article: Hong, Kong. Statistical analysis: Kim. Study supervision: Hong.

\section{Supplemental Information \\ Videos}

Video 1. https://vimeo.com/403269737.

\section{Correspondence}

Chang-Ki Hong: Gangnam Severance Hospital, Yonsei University Health System, Seoul, Republic of Korea. yedamin@yuhs.ac. 